\title{
Multilayered Phantom for Input Impedance Evaluation of Human Body Communication Electrodes
}

\author{
Dairoku Muramatsu \\ Graduate School of Frontier Sciences, The Univ. of Tokyo \\ 5-1-5 Kashiwano-ha, Kashiwa, Chiba 277-8563, JAPAN \\ +81-4-7136-4618, JP \\ muramatsu@ems.k.u-tokyo.ac.jp \\ Kohji Koshiji \\ RIST, Tokyo University of Science \\ 12641 Yamazaki, Noda-shi, Chiba 278-8510, JAPAN \\ +81-4-7124-1501, JP \\ koshiji@rs.noda.tus.ac.jp
}

Fukuro Koshij

Faculty of Engineering, Tokyo Polytechnic University

1583 liyama, Atsugi, Kanagawa, 243-0297, JAPAN

+81-3-3372-1321, JP

koshiji@gen.t-kougei.ac.jp

Ken Sasaki

Graduate School of Frontier Sciences, The Univ. of Tokyo

5-1-5 Kashiwano-ha, Kashiwa, Chiba 277-8563, JAPAN +81-4-7136-4621, JP

ksasaki@k.u-tokyo.ac.jp

\begin{abstract}
This paper presents the input impedance measurement of a human body communication electrode by using newly developed multilayered phantom. Based on the comparison between analysis and measurement values, it was found that the multilayered phantom can be used for accurate evaluation.
\end{abstract}

\section{Keywords}

Human body communication, Biological tissue, Phantom, Electrode, Input impedance

\section{MEASUREMENT AND ANALYSIS}

Human body communication (HBC) is one of the physical layers of IEEE802.15.6, which utilizes a part of the human body as a transmission medium. In HBC, radio frequency signal is excited or received through the electrodes attached to the human body. In order to evaluate the input impedance characteristics of the electrodes (antennas), multilayered tissue structure phantom is needed because the human body consists of many different biological tissues which have different electrical properties [1]. In this paper, the input impedance characteristics of a transmitter electrode were measured by using the multilayered phantom and compared with the analyzed value obtained from a detailed model.

Figure 1 shows the configuration of a multilayered phantom mimicking the human arm and a transmitter. Table 1 shows the relative permittivity and conductivity of the each phantom layer at 10, 20 and $30 \mathrm{MHz}$. These electrical properties were measured by the transmission method using a coaxial line. Figure 2 shows the input impedance characteristics of the transmitter electrode. Solid lines show the value measured by the multilayered phantom. Broken lines show the value analyzed by a detailed human arm model including seven different biological tissues using FDTD method. Circle markers show the value obtained from the FDTD simulation with the phantom model which has the shape shown in
Fig. 1 and electrical properties shown in Table 1.The black and red line shows the real and imaginary part of the input impedance respectively. These differences in the input impedance between the measurement and analysis shown in Fig. 2 are within an acceptable range considering individual differences of real bodies.
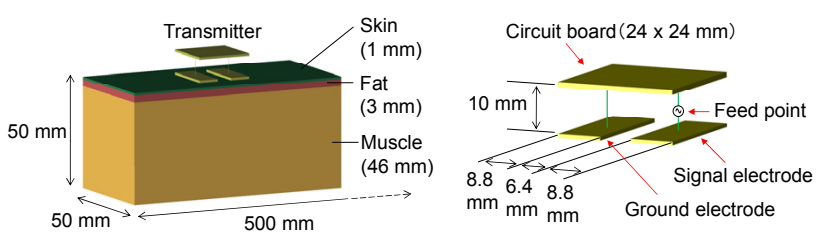

Figure 1. Configuration of phantom and transmitter electrode.

Table 1. Electrical properties of each phantom layer

\begin{tabular}{|l|l|l|l|l|l|l|}
\hline \multirow{2}{*}{ Tissue } & \multicolumn{2}{|c|}{$10 \mathrm{MHz}$} & \multicolumn{2}{c|}{$20 \mathrm{MHz}$} & \multicolumn{2}{c|}{$30 \mathrm{MHz}$} \\
\cline { 2 - 7 } & $\varepsilon_{r}$ & $\sigma[\mathrm{S} / \mathrm{m}]$ & $\varepsilon_{r}$ & $\sigma[\mathrm{S} / \mathrm{m}]$ & $\varepsilon_{r}$ & $\sigma[\mathrm{S} / \mathrm{m}]$ \\
\hline \hline Skin & 421 & 0.17 & 283 & 0.36 & 196 & 0.50 \\
\hline Fat & 71 & 0.028 & 39 & 0.060 & 26 & 0.076 \\
\hline Muscle & 184 & 0.67 & 141 & 0.75 & 111 & 0.78 \\
\hline
\end{tabular}

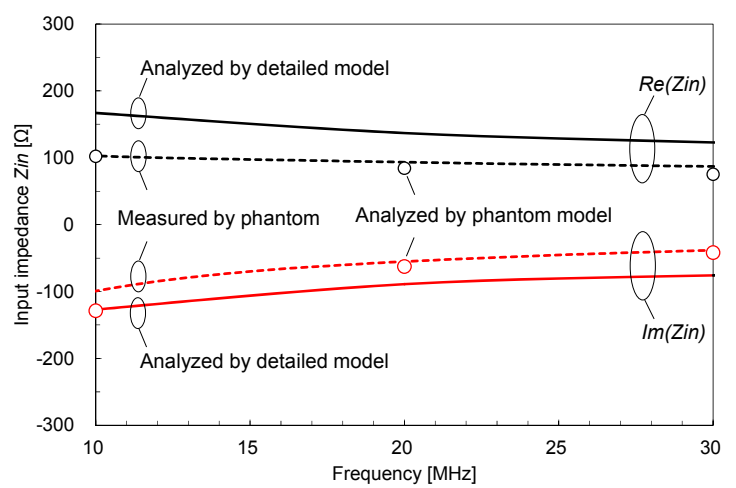

Figure 2. Input impedance characteristics of the electrode.

\section{ACKNOWLEDGMENTS}

A part of this research was carried out through a Grant-in-Aid for JSPS Fellows (25-5924).

\section{REFERENCES}

[1] D. Muramatsu, et al., Trans of JIEP, Vol. 16, No. 7, pp. 528534, 2013. 